\title{
Calcineurin-Responsive Transcription Factor CgCrzA Is Required for Cell Wall Integrity and Infection-Related Morphogenesis in Colletotrichum gloeosporioides
}

\author{
Ping Wang ${ }^{1 \dagger}$, Bing Li ${ }^{2 \dagger}$, Yu-Ting Pan ${ }^{1}$, Yun-Zhao Zhang ${ }^{1}$, De-Wei Li $\mathbb{D}^{3}$, and Lin Huang (D) ${ }^{1 *}$ \\ ${ }^{1}$ Co-Innovation Center for Sustainable Forestry in Southern China, Nanjing Forestry University, Nanjing, Jiangsu \\ 210037, China \\ ${ }^{2}$ College of Horticulture and Plant Protection, Yangzhou University, Yangzhou, Jiangsu 225009, China \\ ${ }^{3}$ The Connecticut Agricultural Experiment Station Valley Laboratory, Windsor, CT 06095, USA
}

(Received on April 23, 2020; Revised on August 25, 2020; Accepted on August 31, 2020)

The ascomycete fungus Colletotrichum gloeosporioides infects a wide range of plant hosts and causes enormous economic losses in the world. The transcription factors (TFs) play an important role in development and pathogenicity of many organisms. In this study, we found that the $\mathrm{C}_{2} \mathrm{H}_{2}$ TF CgCrzA is localized in both cytoplasm and nucleus under standard condition, and it translocated from cytoplasm to nucleus in a calcineurin-dependent manner. Moreover, the $\triangle \mathrm{CgCrz} A$ was hypersensitive to cell wall perturbing agents and showed severe cell wall integrity defects. Deletion of the $C g C R Z A$ inhibited the development of invasive structures and lost pathogenicity to plant hosts. Our results suggested that calcineurin-responsive TF CgCrzA was not only involved in regulating cell wall integrity, but also in morphogenesis and virulence in $C$. gloeosporioides.

Keywords : anamorphic fungus, cell wall integrity, coelo-

\footnotetext{
${ }^{\dagger}$ These authors contributed equally to this work.

*Corresponding author.

Phone) +86-25-85427301

E-mail) lhuang@njfu.edu.cn

ORCID

De-Wei Li

https://orcid.org/0000-0002-2788-7938

Lin Huang

https://orcid.org/0000-0001-7536-0914

(c) This is an Open Access article distributed under the terms of the Creative Commons Attribution Non-Commercial License (http:// creativecommons.org/licenses/by-nc/4.0) which permits unrestricted noncommercial use, distribution, and reproduction in any medium, provided the original work is properly cited.
}

Articles can be freely viewed online at www.ppjonline.org. mycetes, transcription factor, virulence

Handling Editor : Junhyun Jeon

In eukaryotic organisms, transcription factors (TFs) regulate a wide range of physiological processes by binding to specific DNA sites, where activation or repression of gene transcription occurs (Kusuya et al., 2017; Pabo and Sauer, 1992; Yun et al., 2019). Based on the differences of DNA-binding domain types, TFs can be divided into different classes, including basic region leucine zipper (bZIP), MADS-box, myb, helix-loop-helix, homeobox, and zinc fingers (Chen et al., 2002; Pabo and Sauer, 1992). $\mathrm{C}_{2} \mathrm{H}_{2}$ zinc-finger proteins (ZFPs) are a large TF family in eukaryotes (Englbrecht et al., 2004). In nonpathogenic model yeast Saccharomyces cerevisiae, approximately $0.8 \%$ of genes encode $\mathrm{C}_{2} \mathrm{H}_{2}$ TF proteins. They are involved in various aspects of growth, development, and stress responses. Sixty-nine $\mathrm{C}_{2} \mathrm{H}_{2}$ TFs were characterized in the ascomycete pathogen Magnaporthe oryzae, and 22 of them were involved in plant infection (Cao et al., 2016). In another phytopathogen Fusarium graminearum, $91 \mathrm{C}_{2} \mathrm{H}_{2} \mathrm{TFs}$ were identified, and 16 of them were found to be associated with virulence (Son et al., 2011). These reports suggested that the $\mathrm{C}_{2} \mathrm{H}_{2}$ TFs may play important roles in the development, stress resistance, and plant infection in fungi.

The cell wall integrity (CWI) pathway is an essential signal transduction pathway, which is required for the adaptation to various cell wall perturbing conditions of host plants (Geoghegan et al., 2017; Gow et al., 2017). Activation of the CWI pathway is initiated when transmembrane sensors recognize external stress signals. These sensors transfer 
the signal to Rho GTPase, then stimulates downstream effectors that regulate the mitogen-activated protein kinase cascade. The end kinase Slt 2 of the cascade controls downstream TFs, such as Rlm1 and Swi4/6, that trigger the transcription of genes involved in cell wall biogenesis (Levin, 2011). Recent studies identified signaling cross-talk between the CWI pathway and other signaling cascades, such as the calcineurin pathway or the high osmolarity glycerol pathway (Dichtl et al., 2016). In S. cerevisiae, a calcineurin-responsive zinc finger TF Crz1/CrzA, which acts as a major target of calcineurin and is dephosphorylated upon activation of calcineurin and trans-localized from cytosol to nucleus, to regulate downstream gene expression (Cyert, 2003). Crz1 is revealed to contribute to CWI maintenance (Garrett-Engele et al., 1995) through the induced expression of FKS2 and other cell wall synthesis-related genes (García et al., 2004; Stathopoulos and Cyert, 1997; Zhao et al., 1998). In filamentous phytopathogenic fungi, numerous studies have shown that the CRZ1 are homologs required for ion stress resistance, secondary metabolism, virulence, and pathogenicity (Chen et al., 2019; Schumacher et al., 2008). In M. oryzae, the MoCRZ1 deletion mutant showed increased susceptibility to cell wall disturbing agents Congo red (CR) and sodium dodecylsulfate (SDS) (Choi et al., 2009). However, in Valsa pyri, deletion of VpCRZ1 significantly increased mycelial growth and resistance to $\mathrm{CR}$, Calcofluor white (CFW), and SDS and functioned as a negative regulator of cell wall stresses (He et al., 2016). The data suggested that $C R Z 1$ homologs may share different regulation mechanisms of CWI maintenance in different plant pathogenic fungi.

Colletotrichum gloeosporioides is a hemibiotrophic fungal pathogen, which causes anthracnose on a wide range of plant hosts and causes enormous economic losses in the world (Dean et al., 2012; Xu et al., 2016). C. gloeosporioides has emerged as an attractive model for studying both fungal pathogenicity and plant immune response due to the genetic tractability and availability of genomic data (Dean et al., 2012; Huang et al., 2019). Anthracnose caused by C. gloeosporioides is a serious fungal disease on Chinese fir (Cunninghamia lanceolata) and leads to enormous economic losses in China (Huang et al., 2019). Despite its economic and scientific importance, molecular mechanisms of $C$. gloeosporioides are largely unknown. Calcineurinresponsive zinc finger TF CrzA displays multifunctions in regulating growth, stress insistence, and pathogenicity in fungi (Chen et al., 2019; Choi et al., 2009; He et al., 2016). In C. gloeosporioides, knockout of CRZA resulted in detrimental effects on growth, sporulation, germination, and appressorium formation (Dubey et al., 2016). However, whether the localization $C g C R Z A$ is regulated by $\mathrm{Ca}^{2+}$ signaling, and whether $C g C R Z A$ is involved in the CWI pathway is still not clear. Thus, this study aimed to evaluate the extracellular $\mathrm{Ca}^{2+}$ on the localization pattern of CgCrzA. In addition, the functions of $\mathrm{CgCrzA}$ in cell wall stressors' resistance, and infection-related morphogenesis were investigated using a gene disruption strategy. The findings of the present study provide an insight into CWI and molecular mechanisms of pathogenicity in C. gloeosporioides.

\section{Materials and Methods}

Fungal strains and culture conditions. The wild type (WT) C. gloeosporioides s.s. strain SMCG1\#C was used for transformation. The WT, $\Delta C g c r z A$ mutant, and complemented strains were maintained on potato dextrose agar (PDA) medium (Yang et al., 2018). Fungal growth assays were performed on PDA and complete medium (CM) at $25^{\circ} \mathrm{C}$ for 5 days in the dark. For sporulation, strains were cultured in liquid carboxymethyl cellulose (CMC) medium and assayed as previously described (Araújo et al., 2014). $\mathrm{CaCl}_{2}(0.2 \mathrm{M})$, SDS (0.005\%), CFW (200 $\left.\mu \mathrm{g} / \mathrm{ml}\right)$, Congo red $(200 \mu \mathrm{g} / \mathrm{ml})$, and lysing enzyme $(15 \mu \mathrm{g} / \mathrm{ml})$ were added to the $\mathrm{CM}$ agar to determine the various stress resistance of tested strains.

Fungal protoplast preparation and transformation were conducted according to the method described by Li et al. (2019). $\mathrm{TB}_{3}$ agar medium supplemented with $250 \mu \mathrm{g} / \mathrm{ml}$ hygromycin B (Solarbion, Beijing, China) and $400 \mu \mathrm{g} / \mathrm{ml}$ geneticin (MP Biochemical, Santa Ana, CA, USA) was used for the selection of candidate transformants.

Construction of the $C g C R Z A$ knock out and complemented strains. The $C g C R Z A$ gene replacement constructs were established using the double-joint PCR method as described (Yu et al., 2004). The upstream and downstream flanking regions $(\sim 1.5 \mathrm{~kb})$ of $C g C R Z A$ were amplified and fused on either side of the hygromycin B phosphotransferase gene $(H P H)$ cassette to generate the gene replacement constructs. Then, the constructs were transformed into protoplasts of the WT as previously described (Yang et al., 2018). Subsequently, the candidate transformants were screened by PCR analysis of open reading frame of $C g C R Z A$ and further confirmed by Southern blotting. For complementation, a DNA fragment containing the $C g C R Z A$ gene, and its native promoter was amplified and inserted into the vector pYF11 using the yeast gap repair approach (Zhou et al., 2011). The resulting complementation construct $C g C R Z A:: G F P$ was transformed into protoplasts of the $\triangle C g c r z A$ mutant to generate the complement- 
ed transformants. Primers used in this study were listed in the Supplementary Table 1.

\section{Light microscopy observation of localization of} CgCrzA. Fresh mycelia of the complemented transformants were cultured in liquid CM medium at $25^{\circ} \mathrm{C}$ for $24 \mathrm{~h}$ and stained with $10 \mu \mathrm{g} / \mathrm{ml}$ DAPI (Sigma-Aldrich, St. Louis, MO, USA). To evaluate the effect of $\mathrm{CaCl}_{2}$ and FK506 on localization of CgCrzA, $0.2 \mathrm{M} \mathrm{CaCl}_{2}$ or $10 \mu \mathrm{g} / \mathrm{ml} \mathrm{FK506}$ was, respectively, added to liquid $\mathrm{CM}$ medium for $30 \mathrm{~min}$ at $28^{\circ} \mathrm{C}$ before observation. Photographs were taken under a confocal laser scanning microscope (Zeiss, Oberkochen, Germany).

Assays of protoplast release and chitin content. Mycelial plugs of WT were inoculated in liquid CM medium, and cultured for 2 days. Then, mycelia were harvested using two layers of Miracloth (EMD Millipore, Billerica, MA, USA). Mycelia were washed three times and resuspended in $20 \%$ sucrose. Lysing enzyme (Sigma-Aldrich) was added to mycelial suspension $(5 \mathrm{mg} / \mathrm{ml})$. The protoplasts were collected using four layers of Miracloth. The number of protoplasts generated was counted using a hemacytometer under a microscope at 30,60, and 90 min after incubation, respectively. The experiment was conducted three times, and each treatment had three replicates.

Chitin content of mycelia was examined as described by Bulik et al. (2003). Briefly, $5 \mathrm{mg}$ of freeze-dried mycelia was resuspended in $1 \mathrm{ml}$ of $6 \% \mathrm{KOH}$ and heated at $80^{\circ} \mathrm{C}$ for $90 \mathrm{~min}$. Samples were centrifuged at 13,000 rpm for $10 \mathrm{~min}$, and pellets were washed with phosphate buffered saline buffer and resuspended in $0.5 \mathrm{ml}$ of Mcllvaine's buffer ( $\mathrm{pH}$ 6.0). One hundred $\mathrm{ml}$ (13 units) of Streptomyces plicatus chitinase (Sigma-Aldrich) was added, and the mixture was incubated at $37^{\circ} \mathrm{C}$ for $16 \mathrm{~h}$. Then, $100 \mathrm{ml}$ of 0.27 $\mathrm{M}$ sodium borate ( $\mathrm{pH}$ 9.0) was added, and the mixture was heated for $10 \mathrm{~min}$ at $100^{\circ} \mathrm{C}$ with a final addition of $1 \mathrm{ml}$ of diluted Ehrlich's reagent. After incubation at $37^{\circ} \mathrm{C}$ for $20 \mathrm{~min}, 1 \mathrm{ml}$ of the final mixture was used to measure the $\mathrm{OD}_{585 \mathrm{~nm}}$ absorbance value. Standard curves were prepared with GlcNAc (Sigma-Aldrich). The experiment was conducted three times, and each treatment had three replicates.

Assays for sporulation and appressorial turgor pressure. For sporulation, mycelial plugs (5 $\mathrm{mm}$ in diameter) of each isolate were taken from the growing margins of colonies grown on PDA for 5 days and inoculated in liquid $\mathrm{CMC}$ medium and the culture was shaken at $150 \mathrm{rpm}$ for $48 \mathrm{~h}$ at $25^{\circ} \mathrm{C}$. Conidial germination assays were conducted according to the methods described by Fang et al. (2018).
The experiment was conducted three times, and each treatment had three replicates.

For appressorium formation assays, $20-\mu 1$ drops of conidial suspensions were placed on glass coverslips (Fisher Scientific, St. Louis, MO, USA) and incubated at $25^{\circ} \mathrm{C}$. After $4 \mathrm{~h}$ and $12 \mathrm{~h}$ post-incubation, the percentages of conidial germination and appressorial formation were determined under a compound microscope. Appressorium turgor pressure was examined by incipient cytorrhysis assay using 1 to $4 \mathrm{M}$ of glycerol solution, as previously described by Foster et al. (2003). The experiment was conducted three times, and at least 100 conidia per replicate.

Penetration and pathogenicity analysis. The onion penetration assays was performed following the method described by Xu et al. (1998). Onion epidermal strips were prepared and $20 \mu \mathrm{l}$ of conidial suspensions were inoculated onto the surface to induce invasive hyphae (IH) development. After $24 \mathrm{~h}$ post inoculation, IH were observed under a Zeiss Axio Imager A2m microscope (Carl Zeiss, Jena, Germany). IH were divided into four different types (type I, no hyphal penetration; type II, IH with one branch; type III, $\mathrm{IH}$ with at least two branches, but the IH are less extended; type IV, IH with numerous branches and extensive hyphal growth). The experiment was conducted three times, and at least 30 invasive structures were observed for each treatment.

Healthy detached leaves or tissue culture seedlings of Cunninghamia lanceolata, Populus $\times$ euramericana $\mathrm{cv}$. 'Nanlin895' and Liriodendron chinensis $\times$ tulipifera were used to evaluate the pathogenicity of the tested fungal isolates. Conidial suspensions were adjusted to a final concentration of $1 \times 10^{5}$ spores $/ \mathrm{ml}$. Five $\mu \mathrm{l}$ of conidial suspensions were inoculated onto the wounded and unwounded leaves of the aforementioned plants. The inoculated leaves and plants were kept in a moist chamber at $25^{\circ} \mathrm{C}$. Five days post inoculation, the disease severity was assessed. This experiment conducted three times, and each treatment had at least six replicates.

Quantitative real-time PCR (qRT-PCR) and statistical analysis. Total RNA was extracted from fungal mycelia using the TRIzol LS reagent (Invitrogen, Carlsbad, CA, USA). One microgram of the RNA was reverse transcribed into the first-strand cDNA according to the manual of HiScript II Reverse Transcriptase (Vazyme Biotech Co., Nanjing, China), and the cDNA was used as the templates of qRT-PCR. qRT-PCR reactions were conducted following the method described by Yang et al. (2018), and 18S rRNA was used as a reference gene. The experiment was 
performed three times, and each treatment had three replicates.

In all experiments, the mean \pm standard deviation (SD) were calculated for each treatment. Significant differences among treatments were statistically analyzed using oneway analysis of variance (ANOVA) followed by least significant difference's multiple range tests. Student's $t$ test was used to assess the difference of the chitin synthase expression level, and collapsed appressorial ratios between the WT and $\triangle C g c r z A$ mutant, respectively. Statistical analyses were performed using the SPSS version 2.0 software package (SPSS Inc., Chicago, IL, USA).

\section{Results}

CgCrzA translocalization from cytoplasm to nuclei depending on calcineurin. An orthologue of Crz1, CgCrzA (GenBank accession no. APU53507) has been identified in the C. gloeosporioides, which contained two zinc finger motifs at the C-terminus and showed a high similarity to it orthologs in M. oryzae and F. graminearum (Supplementary Fig. 1A and B).

A gene deletion mutant $\triangle C g c r z A$ was generated by replacing the $C g C R Z A$ with a $H P H$ cassette in this study (Fig. 1A). Southern blot assays revealed that the $C g C R Z A$ had only one copy and was deleted in the $\triangle C g c r z A$ mutant (Fig. 1B). The mutant was complemented by reintroducing a $G F P$ fusion construct. In the complemented strain $\triangle C g c r z A / C g C R Z A$, strong GFP signals of the fusion protein were observed in both of the nuclei and cytoplasm in vegetative hyphae (Fig. 2A). However, after the complemented strain was treated with $0.2 \mathrm{M} \mathrm{CaCl}_{2}$ for $30 \mathrm{~min}$,
A

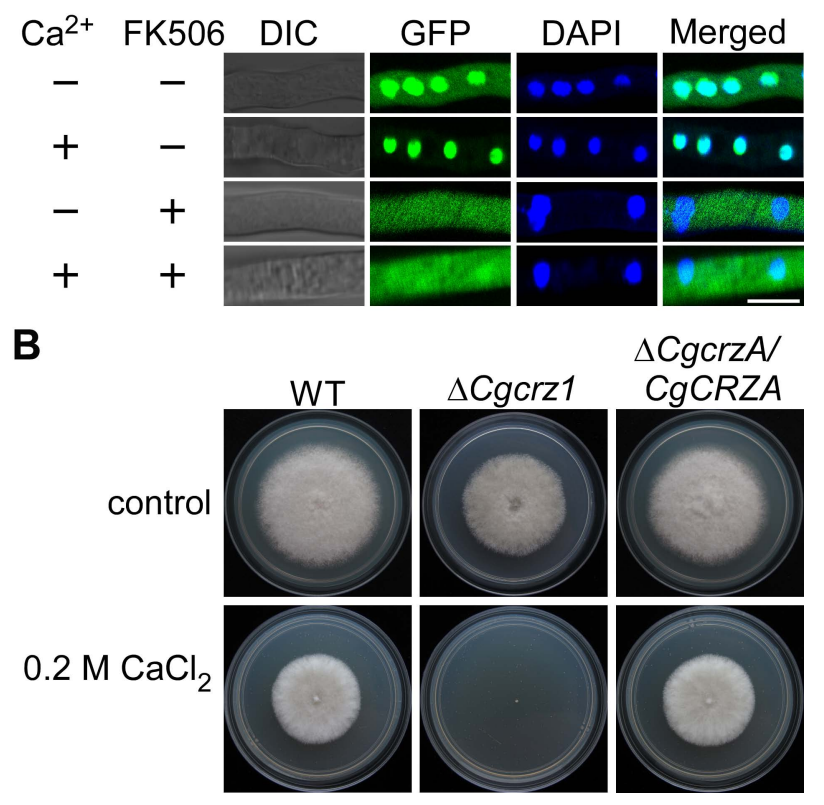

Fig. 2. Translocation of $C g C r z A$ to nucleus regulated by calcineurin. (A) GFP signals in the hyphae of the transformant expressing the $C g C R Z A-G F P$ fusion construct. Epifluorescence observation of the hyphae untreated or treated with $0.2 \mathrm{M} \mathrm{CaCl}_{2}$ or calcineurin inhibitor FK506. Scale bar $=10 \mu \mathrm{m}$. (B) Resistance of wildtype, $\Delta C g c r z A$ mutant and complemented strains to $0.2 \mathrm{M} \mathrm{CaCl}_{2}$ stress.

CgCrzA-GFP signals were translocated from the cytosol to nucleus, and almost no GFP signal was observed in the cytoplasm. Addition of the calcineurin inhibitor FK506 (FK506-binding protein complex FKBP12 that binds to calcineurin) completely blocked nuclear accumulation
A

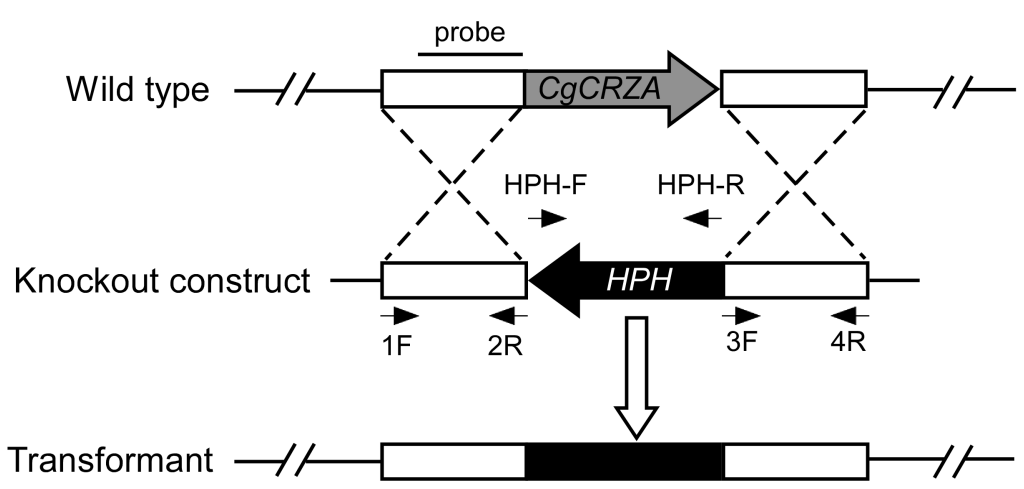

B

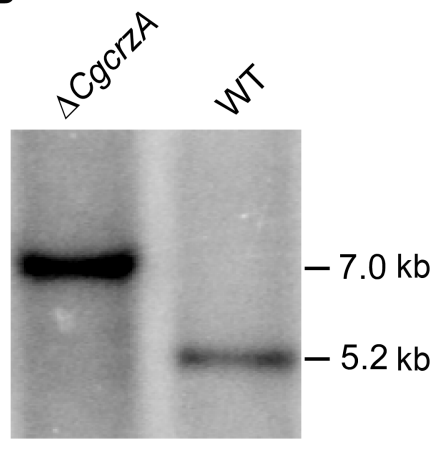

Fig. 1. $C g C R Z A$ knockout vector construction and identification of gene deletion mutant. (A) The $C g C R Z A$ knockout fragment was constructed by PCR amplification. The $c a$. 1.5-kb upstream and downstream flanking sequences were amplified with primers $1 \mathrm{~F} / 2 \mathrm{R}$ and 3F/4R, respectively, and over-lap with the hygromycin B phosphotransferase gene $(H P H)$ cassette. (B) Southern blot analysis of wild type and $\triangle$ CgcrzA mutant. Genomic DNA of wild type and the deletion mutant was extracted and digested with Xho I. The upstream flanking region of $C g C R Z A$ was amplified with primer set CRZA-probe-1F/2R and used as a probe for Southern blot analysis. 
A
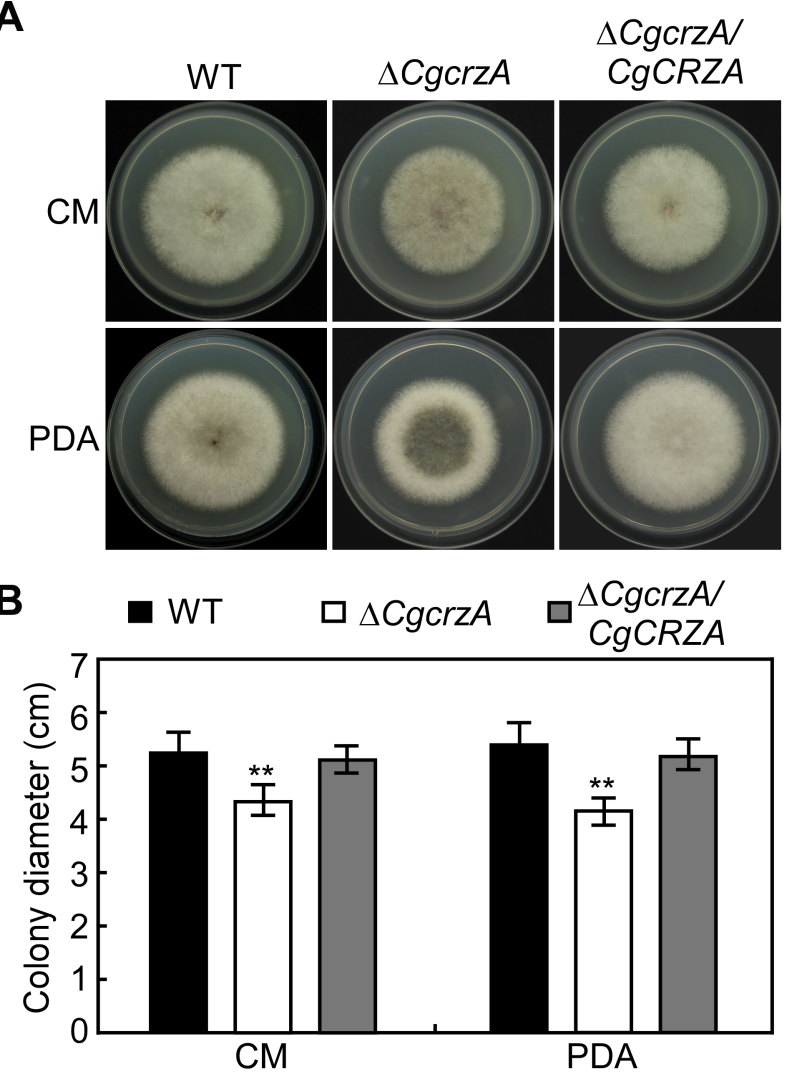

C

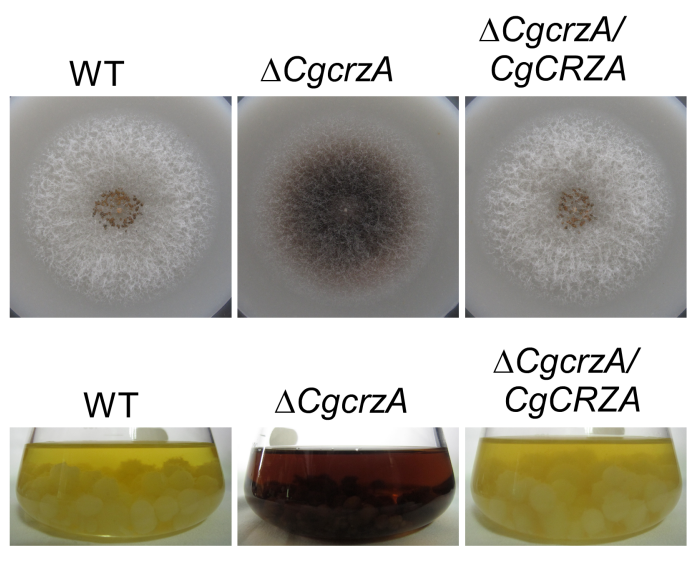

E

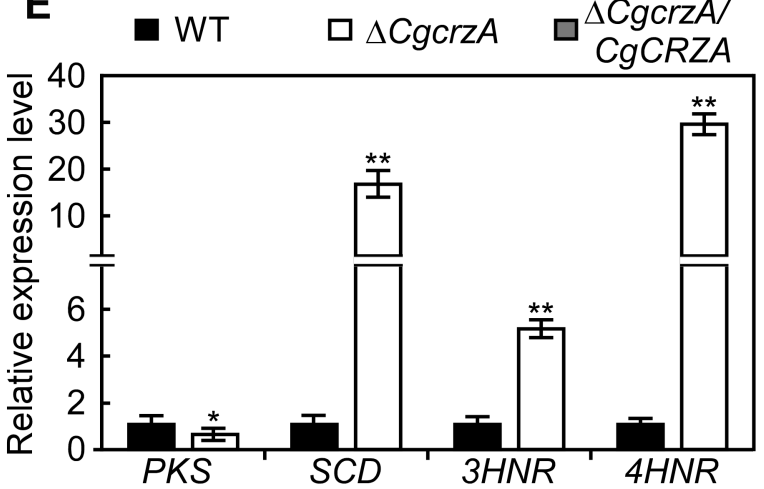

Fig. 3. Effects of $C g C R Z A$ deletion on aerial hyphal growth and melanization. (A) Colonies of wild type (WT), $\triangle C g c r z A$ mutant and the complemented strain $\triangle C g c r z A / C g C R Z A$ were cultured on complete medium $(\mathrm{CM})$ and potato dextrose medium at $25^{\circ} \mathrm{C}$ for 5 days. (B) Colony diameter in (A), the data represent three replicates. (C, D) Colony melanization increased in the $\triangle C g c r z A$ mutant. The tested strains were cultured on oatmeal agar medium and liquid CM medium, respectively. Asterisks indicate significant difference among different treatments at $P<0.01$. (E) Expression level of four melanin biosynthesis genes ( $P K S, S C D, 3 H N R$, and $4 H N R)$. Asterisks indicate significant difference among different treatments at $P<0.05(*)$ or $0.01(* *)$.

of GFP signals (Fig. 2A). In addition, vegetative growth of the $\triangle C g c r z A$ mutant almost was completely inhibited compared with the WT and complemented strains when inoculated on the PDA plates containing $0.2 \mathrm{M} \mathrm{CaCl}_{2}$ (Fig. 2B). These data indicated that activation of calcineurin by exogenous $\mathrm{Ca}^{2+}$ was involved in regulating translocation of $\mathrm{CgCrzA}$ from the cytoplasm to the nucleus.

Involvement of $\mathrm{CgCrzA}$ in vegetative growth and colony melanization. Five days after growing on $\mathrm{CM}$ and PDA plates, the $\triangle C g c r z A$ mutant showed a decreased radial growth than those of the WT and complemented strains $\triangle C g c r z A / C g C R Z A$ (Fig. 3A and B). Interestingly, compared with the WT and complemented strains, the colony melanin of the $\triangle C g c r z A$ mutant was significantly increased when they were grown on oatmeal agar (OMA) plate (Fig. 3C). Abundant melanin in the $\triangle C g c r z A$ mutant was also observed in liquid CM medium (Fig. 3D). qRT-PCR assays showed that the expression level of four melanin biosynthetic-related genes were significantly different in WT and the $\Delta C g c r z A$ mutant. The expression of polyketide synthase (PKS) was significant decreased in the $\triangle C g c r z A$ mutant. However, the expression levels of $S C D, 3 H N R$, and $4 H N R$ in the $\triangle C g c r z A$ mutant were significantly higher than those of WT (Fig. 3E). These results indicated that CgCrzA was required for regulation of melanization in C. gloeosporioides.

CgCrzA required for CWI. When exposed to the cell wall perturbing agents $\mathrm{CFW}$ and $\mathrm{CR}$, inhibition on mycelial growth of the $\triangle C g c r z A$ mutant was significantly higher than those of the WT and complemented strains, respectively (Fig. 4A and B). Mycelia of the $\triangle C g c r z A$ mutant were even completely inhibited when growing onto PDA plates, where $0.005 \%$ SDS was added (Fig. $4 \mathrm{~A}$ and B). The $\triangle C g c r z A$ mutant was also more sensitive to the lys- 
A

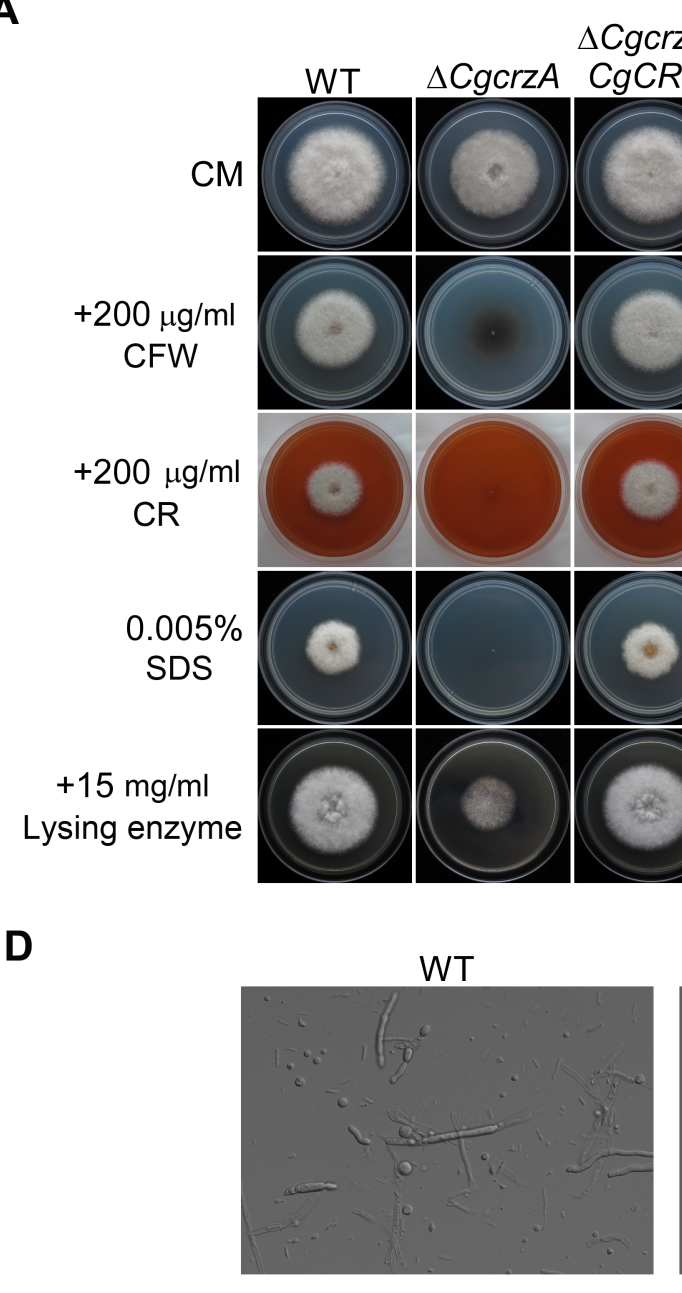

$\mathbf{E}$

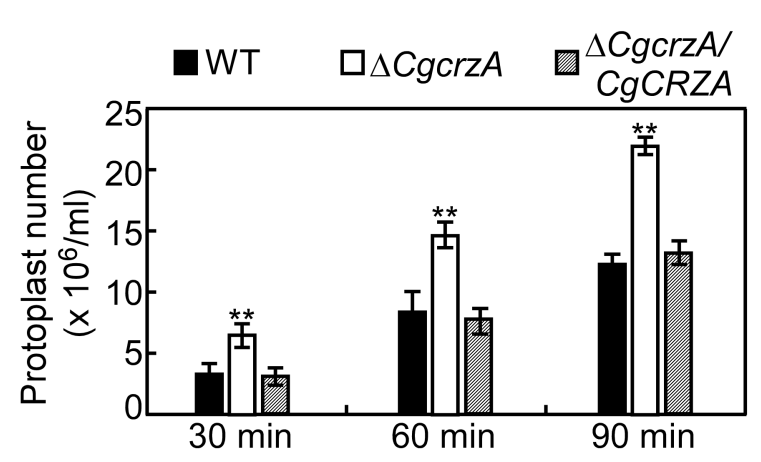

B

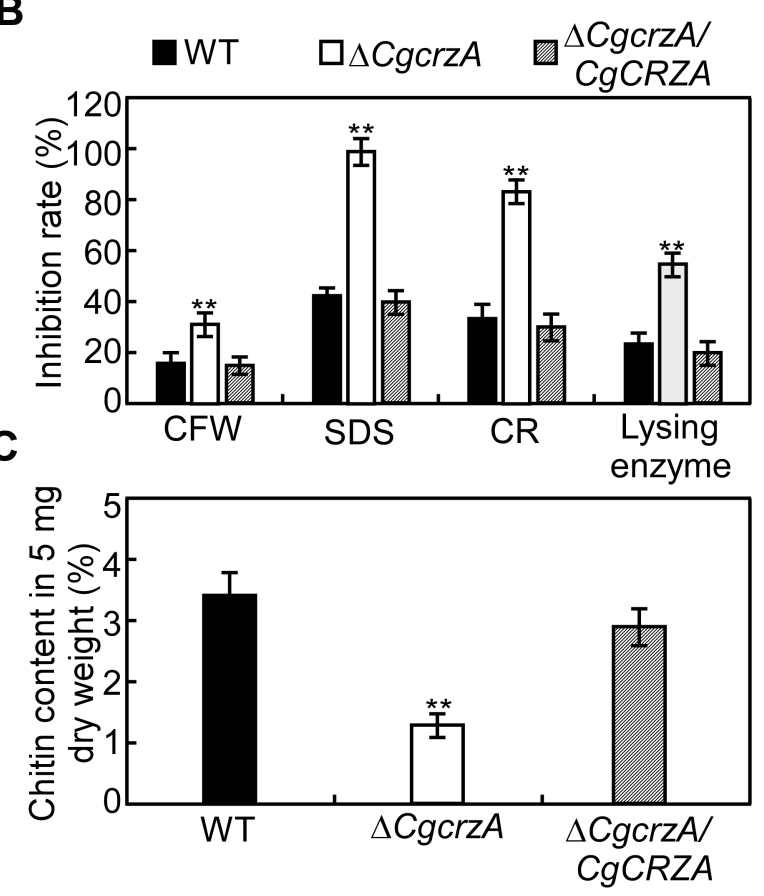

$\triangle \operatorname{CgcrzA}$

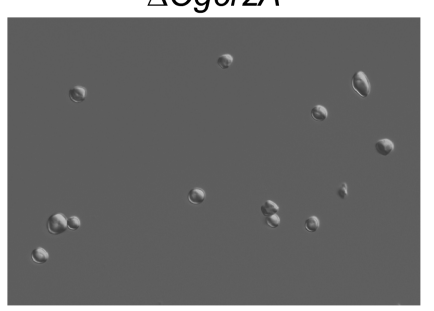

$\triangle \operatorname{Cg} \mathrm{Cr} A / \operatorname{CgCRZA}$

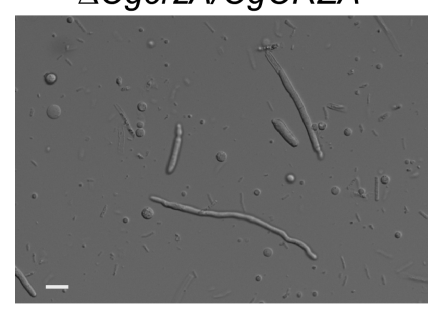

$\mathbf{F}$

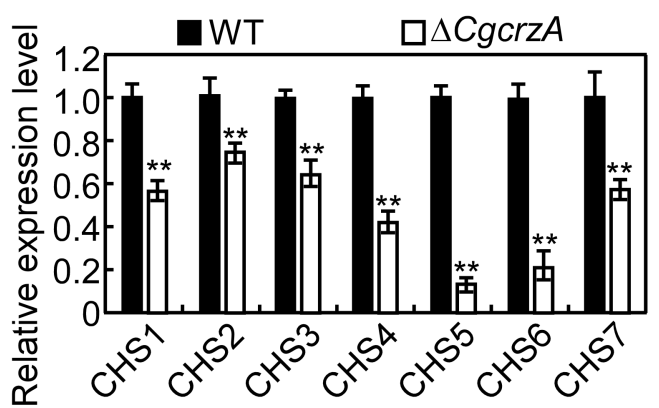

Fig. 4. Deficiency of cell wall integrity in $\triangle C g c r z A$ mutant. (A) Colonies of the wild-type (WT), $\triangle C g c r z A$ mutant and complemented strains were cultured on complete medium (CM) medium supplemented with different cell wall antagonists for 5 days. CFW, Calcofluor white; CR, Congo red; SDS, sodium dodecylsulfate. (B) Colony growth inhibition rate of the indicated strains exposed to various stresses. (C) Mycelial chitin content of the WT, $\triangle$ CgcrzA mutant and complemented strains. (D) Protoplast release after treatment with cell wall-degrading enzymes for $60 \mathrm{~min}$ at $30^{\circ} \mathrm{C}$. Scale bar $=10 \mu \mathrm{m}$. (E) Protoplast numbers at different time points following treatment by cell wall-degrading enzymes. Asterisks indicate significant difference at $P<0.01$. (F) Transcription analysis of seven chitin synthase genes in the wild type and $\triangle C g c r z A$ mutant using quantitative reverse transcription-polymerase chain reaction. Error bars represent the $\mathrm{SD}$ and asterisks indicate significant difference at ${ }^{* *} P<0.01$. 
A

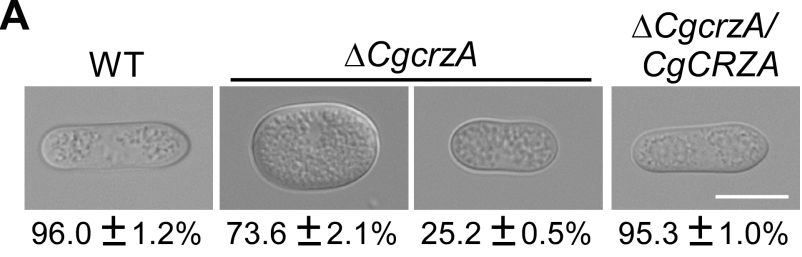

\section{B}

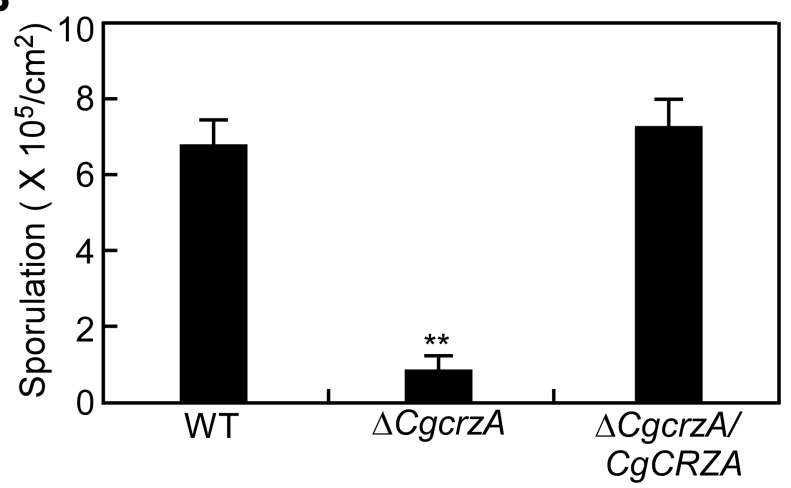

Fig. 5. Effect of $C g C R Z A$ deletion on conidiogenesis. (A) Conidial morphology of the wild type (WT), $\triangle$ CgcrzA mutant and complemented strains. Scale bar $=10 \mu \mathrm{m}$. (B) The number of conidia developed by the WT, $\triangle$ CgcrzA mutant, and complemented strains. Asterisks indicate significant difference at $* * P<0.01$. ing enzyme, which degrades the target chitin in fungal cell wall. Based on these data, we speculated that the $\triangle \operatorname{CgcrzA}$ mutant possibly altered the content of chitin in cell wall, which caused a stronger sensitivity to cell wall inhibitors. To confirm this hypothesis, we further measured the cell wall chitin content of the $\triangle C g c r z A$ mutant. Data showed the mutant had a lower chitin content in mycelia than the WT and complemented strains (Fig. 4C). In addition, we also found that the $\triangle C g c r z A$ mutant released protoplasts more quickly than the WT and complemented strains when they were treated under the same concentration of fungal cell wall chitinase (Fig. 4D and E). Data also showed that the expression level of seven chitin synthase genes was significantly lower in the $\triangle C g c r z A$ mutant than those of the WT (Fig. 4F). According to the aforementioned results, we concluded that $\mathrm{CgCrzA}$ is involved in regulating $\mathrm{CWI}$ in $C$. gloeosporioides.

CgCrzA involved in sporulation and conidial morphology. The morphological observation showed that the percentage of abnormal conidia exceeded $95 \%$ in the $\triangle \mathrm{Cgcrz} A$ mutant compared with $<5 \%$ in the WT and complemented strains (Fig. 5A). Conidial counts also revealed that the co-
A

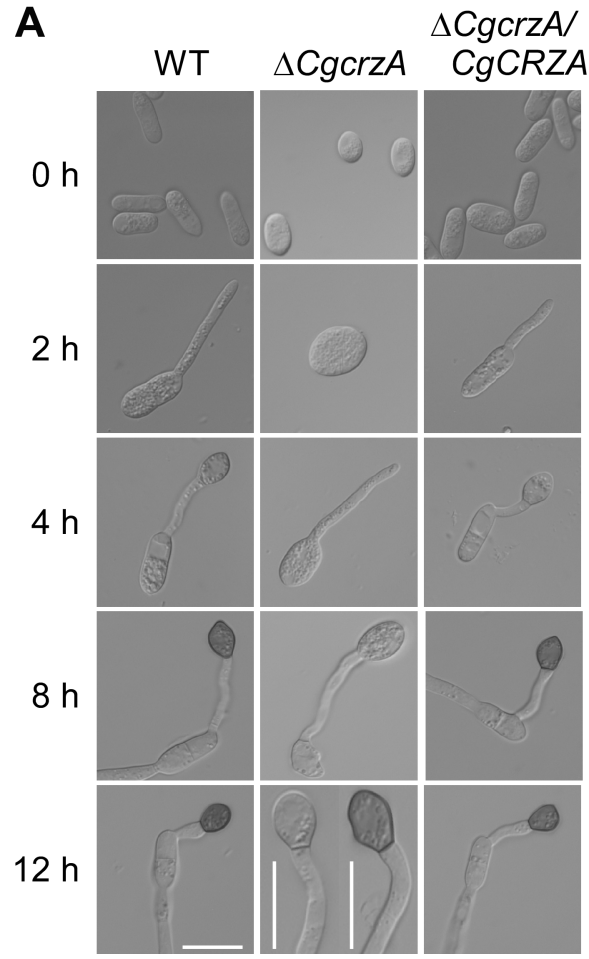

B

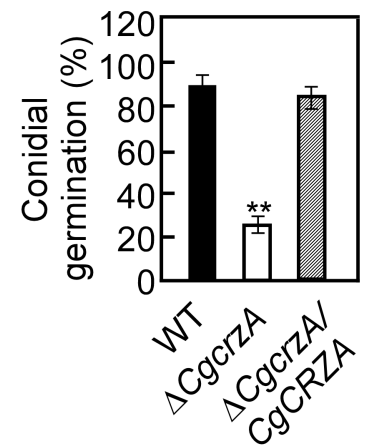

D

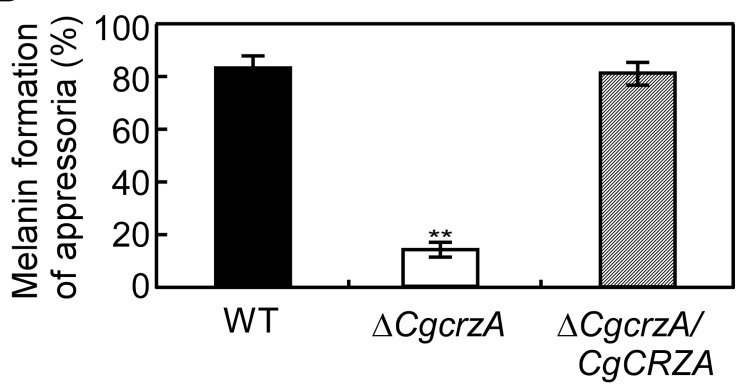

Fig. 6. Defects of conidial germination, appressorial development and melanin accumulation in $\triangle C g c r z A$ mutant. (A) Appressorial development progress of the wild type (WT), $\Delta C g c r z A$ mutant and the complemented strains. Scale bars $=10 \mu \mathrm{m}$. (B) Conidial germination rates at different time intervals in (A). (C) Appressorial formation rates in (A). (D) Melanin formation rates of the appressoria in (A). Error bars represent the SD and asterisks indicate significant difference at $* * P<0.01$. 
A
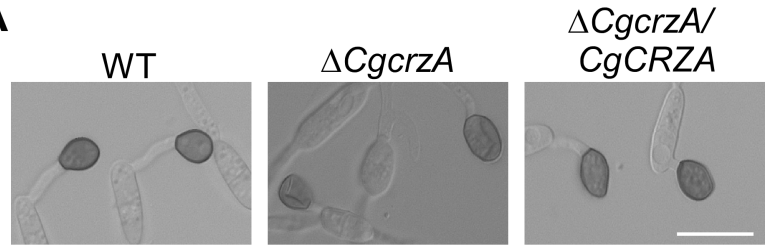

B

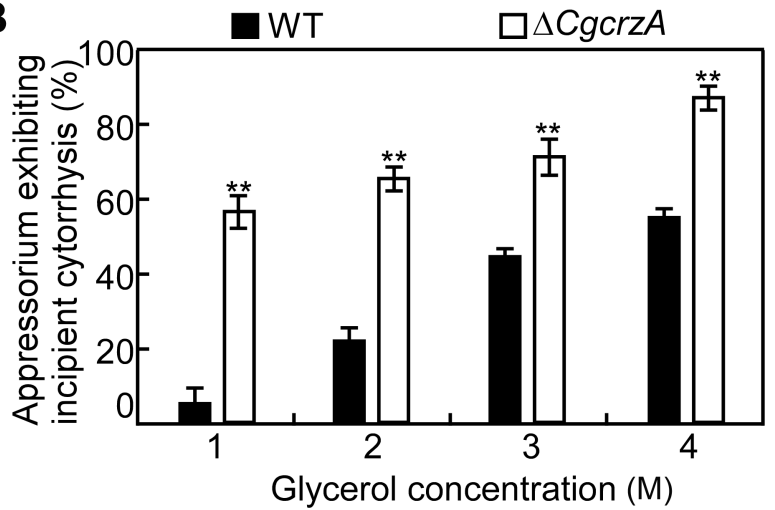

Fig. 7. Defect of appressorial turgor pressure in $\triangle C g c r z A$ mutant. (A) Appressorial morphology of the wild type (WT), $\triangle C g c r z A$ mutant, and complemented strains after treatment by $4 \mathrm{M}$ glycerol solution for $10 \mathrm{~min}$. Scale bar $=10 \mu \mathrm{m}$. (B) Collapsed appressoria of the WT and $\triangle C g c r z A$ mutant. Error bars represent the SD and asterisks indicate significant difference at ${ }^{* *} P<0.01$.

nidia developed by the $\triangle C g c r z A$ mutant were significantly less than those of the WT and complemented strains (Fig. 5B). Compared with the WT and complemented strains, deletion of $C g C R Z A$ significantly inhibited conidial germination (Fig. 6A and B). These data indicated that $\mathrm{CgCrzA}$ is essential for asexual reproduction of $C$. gloeosporioides.

A

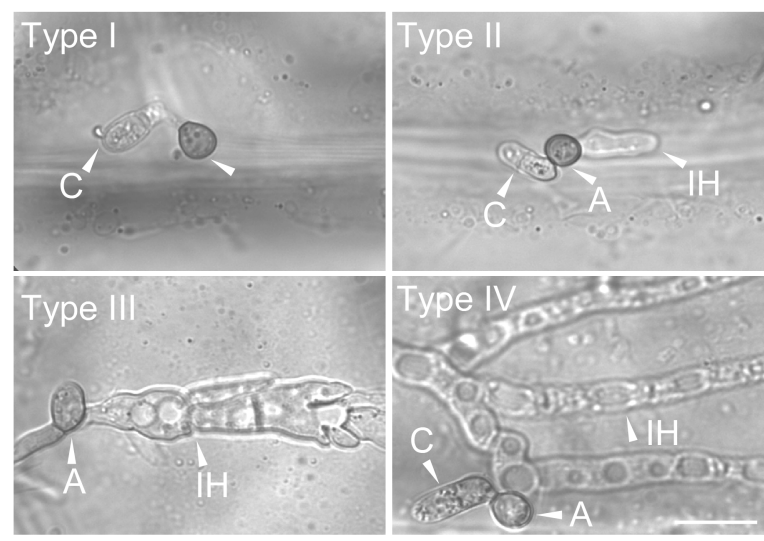

CgCrzA required for invasive structures development and pathogenicity. Appressorial formation rate of the $\triangle C g c r z A$ mutant was considerably reduced than those of the WT and complemented strains (Fig. 6A and C). In the $\triangle C g c r z A$ mutant, over $80 \%$ of appressoria failed to melanize, while the WT and the complemented strains formed normal melanized appressoria (Fig. 6A and D). Compared with the WT, the $\triangle C g c r z A$ mutant showed a higher collapsed appressorial ratio in glycerol solutions, indicating a significantly reduced appressorial turgor pressure (Fig. 7A and B).

Onion epidermis penetration assays showed most of the IH of the WT and complemented strains belonged to type IV, which showed numerous branches and extensive hyphal growth. By contrast, only $10 \%$ of IH belonged to the type III, which showed at least two branches and less extensive hyphae, and type IV in the $\triangle C g c r z A$ mutant (Fig. $8 \mathrm{~A}$ and $\mathrm{B}$ ). These data indicated that $\mathrm{CgCrzA}$ was required for appressorial mature and invasive hyphal development in C. gloeosporioides.

Pathogenicity test results showed that the $\triangle \operatorname{CgcrzA}$ mutant fully lost its pathogenicity on the unwounded leaves of $\mathrm{Cu}$. lanceolata, while the WT developed typical necrotic lesions. This defect in $\triangle C g c r z A$ mutant was completely recovered in the complemented strain (Fig. 9A). Similarly, the $\triangle C g c r z A$ mutant was nonpathogenic on detached leaves of Populus $\times$ euramericana cv. Nanlin895 and Liriodendron chinensis $\times$ tulipifera (Fig. 9B and C). Pathogenicity assays were also performed on wounded leaves of $\mathrm{Cu}$. lanceolata. Result showed the $\triangle C g c r z A$ mutant was unable to cause apparent typical enlaged necrotic lesions like WT and the complemented strain on wounded leave of the plant

\section{B}

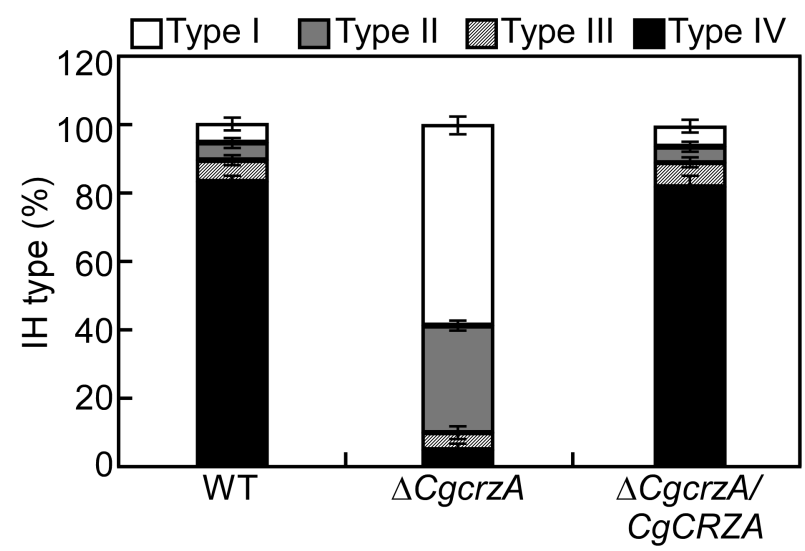

Fig. 8. Effect of $C g C R Z A$ deletion on invasive hyphal development. (A) Morphology of different types of invasive hyphae developed on onion epidermal cells. (B) Ratios of different categories of invasive hyphae (IH) in the wild type (WT), $\triangle C g c r z A$ mutant, and complemented strians. Error bars represent the SD. Scale bar $=10 \mu \mathrm{m}$. A, appressoria; C, conidia. 
A

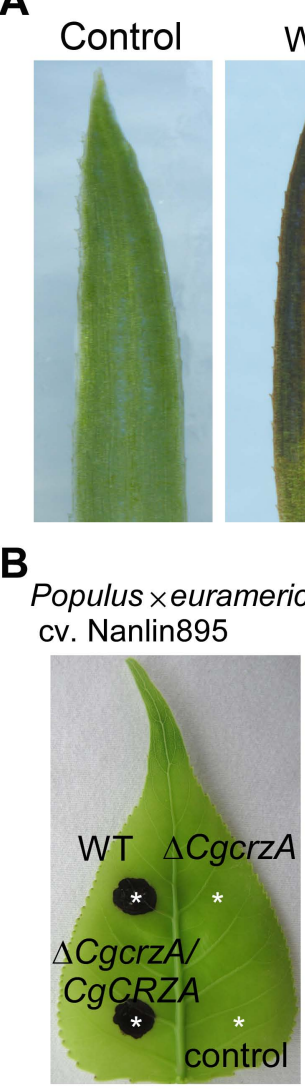

D

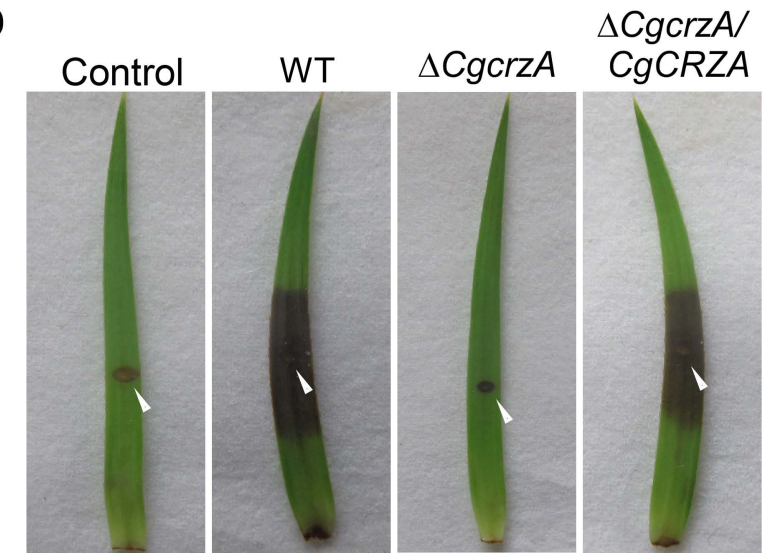

Fig. 9. Pathogenicity of $C g C R Z A$ deletion mutant on host plants. Plant infection assays on leaves of Cunninghamia lanceolata (A), Populus $\times$ euramericana cv. Nanlin895 (B), and Liriodendron chinensis $\times$ tulipifera $(\mathrm{C})$ were inoculated with conidial suspension of wild type, $\triangle C g c r z A$ and the complemented strains $\triangle C g c r z A / C g C R Z A$. $\mathrm{ddH}_{2} \mathrm{O}$ used as the negative control. Diseased leaves were harvested and photographed 5 days after inoculation. Asterisks indicate inoculation sites. (D) Infection assays on wounded leaves of $\mathrm{Cu}$. lanceolata were inoculated with conidial suspension of wild type, $\triangle C g c r z A$ and the complemented strain $\triangle C g c r z A / C g C R Z A$. $\mathrm{ddH}_{2} \mathrm{O}$ used as the negative control. Diseased leaves were harvested and photographed 5 days after inoculation. Arrowheads indicate inoculation sites. host (Fig. 9D). These results indicated that $C g C R Z A$ was required for pathogenicity.

\section{Discussion}

$\mathrm{C}_{2} \mathrm{H}_{2}$ ZFPs comprise of an abundant nucleic acid-binding protein family in the genomes of eukaryotes (Schumacher et al., 2004). $\mathrm{C}_{2} \mathrm{H}_{2}$ ZFPs display a variety of functions, which not only participate in transcriptional regulation of target genes but also act in other cellular functions that are probably associated with the zinc-finger domain (Englbrecht et al., 2004; Foster et al., 2003; Yun et al., 2019). In this study, we found that the calcineurin-responsive TF $C g C r z A$ played important roles in vegetative growth, asexual reproduction, pathogenesis, and also CWI.

In $S$. cerevisiae, Crzlp is localized in the cytosol under standard conditions. When yeast cells were incubated with $\mathrm{Ca}^{2+}$, the localization pattern of Crzp1 was changed from the cytosol to nucleus. This translocation of Crzlp activated calcineurin-dependent transcription (Park et al., 2019). Interestingly, we found that $\mathrm{CgCrzA}$ was localized in both of the cytoplasm and nucleus under standard condition. However, its localization could be translocated from the cytoplasm to the nucleus when the exogenous $\mathrm{Ca}^{2+}$ was added, and this movement was blocked by the addition of calcineurin inhibitor FK506. This difference may lead to some distinct functions and corresponding regulation mechanisms of CgCrzA in C. gloeosporioides. The previous study showed that lower level of $\mathrm{Ca}^{2+}\left(10 \mathrm{mM} \mathrm{CaCl}_{2}\right)$ partially restored the defects of CRZA deletion mutant in growth, sporulation, and appressorial development in $C$. gloeosporioides (Dubey et al., 2016). Our study showed a higher $\mathrm{Ca}^{2+}$ concentration $\left(0.2 \mathrm{M} \mathrm{CaCl}_{2}\right)$ displayed a strong inhibition on vegetative growth of the $\triangle C g c r z A$ mutant. In both M. oryzae and Fusarium graminearum, CRZ1 deletion mutants also displayed strong sensitivity to $\mathrm{CaCl}_{2}$ stress (Choi et al., 2009; Pabo and Sauer, 1992). These data suggested that the localization pattern of $\mathrm{CgCrzA}$ was possibly regulated by calcineurin and exogenous $\mathrm{Ca}^{2+}$ in $C$. gloeosporioides, and this mechanism was shared in different fungi.

Fungal melanin is produced and deposited in cell wall to protecting fungal cells from hydrogen peroxide, UV radiation, and antimicrobial peptides (Doering et al., 1999). In fungi, two strategies are employed to produce melanin. In the first strategy, melanin is synthesized via L-3,4dihyroxyphenylalanine (L-dopa). In the second one, fungi synthesize melanin via the 1,8-dihydroxynaphthalene pathway, which is found in most fungi (Eisenman and Casadevall, 2012). In this pathway, melanin synthesis is 
catalyzed by multiple enzymes including PKS, 1,3,6,8-tetrahydroxynaphthalene reductase (4HNR), 1,3,8-trihydroxynaphthalene reductase (3HNR), and scytalone dehydratase (SCD) (Thompson et al., 2000). Deletion mutants of these genes resulted in defects of melanin accumulation in hyphae and appressoria, and significantly reduced virulence in pathogenic fungi (Schumacher, 2016; Thompson et al., 2000; Wang et al., 2020; Yu et al. 2015). In C. gloeosporioides, the CgSCD1 deletion mutants were unable to accumulate melanin in appressoria and vegetative hyphae, and showed decreased virulence (Wang et al., 2020). In this study, $C g C R Z A$ deletion mutant showed a significantly increased melanin in vegetative growth stage. Genes required for melanin synthesis were differentially expressed at significant levels. In particular, several genes including $\mathrm{SCD}, 3 \mathrm{HNR}$, and $4 \mathrm{HNR}$ were up-regulated specifically in the $\triangle C g c r z A$ mutant. However, deletion of $C g C R Z A$ significantly reduced the expression level of $P K S$, which showed a positive regulation in melanin synthesis in other fungi (Eisenman and Casadevall, 2012; Schumacher, 2016). The data indicates that $P K S$ may share a different regulation mechanism in C. gloeosporioides. Considering the above results, we conclude that $\mathrm{CgCrzA}$ negatively regulates hyphal melanin accumulation in C. gloeosporioides. It should be pointed out that melanin deposition was decreased in most appressoria, which was not consistent with the significant increase of melanin in hyphae. Whether there is a different melanin accumulation regulation between vegetative hyphae and appressoria need more investigations in the future.

Chitin is a key component of the filamentous fungal cell wall and plays important roles in the maintenance of hyphal morphology and function (Lenardon et al., 2010). Recently, chitin has also emerged as a significant player to modify the host's immune response to fungi (Pusztahelyi, 2018). Chitin synthesis is determined by the activity of chitin synthases (Langner and Göhre, 2016). Some chitin synthases have been identified to involve in the interaction with the host plants. For example, the chitin synthases BcChs1 and $\mathrm{BcChs} 3$ are important for chitin synthesis and virulence in Botrytis cinerea. The chitin synthases FgChs2 and FgChs5 are required for pathogenicity in $F$. graminearum (Madrid et al., 2003; Martin-Udiroz et al., 2004; Soulié et al., 2003, 2006). In this study, the $\triangle C g c r z A$ mutant showed reduced growth and hyphal chitin content. Also, the mutant was more sensitive to cell wall perturbing agents and displayed a faster protoplast release than the WT and complemented strains. The transcriptional level of seven chitin synthases was down-regulated in the $\triangle C g c r z A$ mutant, indicating that CgCrzA-mediated chitin synthase activity might be partial- ly responsible for the pathogenicity. However, whether the transcript factor CgCrzA could directly bind the promoter regions of these chitin synthase genes, and its potential binding sites need to be explored in further research.

Previous studies showed that fungi had evolved regulatory networks to ensure the correct spatial and timing of development events (Kim et al., 2010). Hyphal development and conidiogenesis are key steps in the colonization of host plants by many fungal pathogens, and TFs play important regulatory roles in these developmental processes (Kim et al., 2010). We found that the $\Delta C g c r z A$ mutant had defects in vegetative hyphal growth and sporulation, which were consistent with a previous study (Dubey et al., 2016). Furthermore, deletion of CgCRZA altered the normal conidial morphological characters. In $B$. cinerea, the $\Delta B c c r z 1$ mutant also showed impeded hyphal growth and abnormal conidiogenesis (Schumacher et al., 2008). Deletion of CRZ1 in Aspergillus fumigatus and M. oryzae resulted in a similar defect in sporulation as well (Choi et al., 2009; Soriani et al., 2008). These data suggested that $\mathrm{CgCrzA}$ and its orthologs share similar roles in vegetative growth and conidiogenesis in phytopathogenic fungi.

Plant infection of $C$. gloeosporioides was mediated by appressoria (Fang et al., 2018). In phytopathogenic fungi, appressoria accumulate compatible solutes such as glycerol to increase the internal turgor pressure, which produces a mechanical force to directly penetrate the epidermis (Wilson and Talbot, 2009). In the mature stage of appressorium, as an impermeable barrier to osmolytes, melanin layer is formed between the appressorial cell wall and plasma membrane, which is beneficial to the accumulation of high turgor pressure (Ryder et al., 2013). Appressorial formation and melanin deficiencies result in reduced turgor pressure and decreased host infection ability (Chang et al., 2014; Wilson and Talbot, 2009). In a previous study, deletion of $C g C R Z A$ will considerably inhibit the appressorial formation of $C$. gloeosporioides. Our data not only confirmed the involvement of $\mathrm{CgCrzA}$ in appressorial formation, but also showed that $\mathrm{CgCrzA}$ was required for melanin pigmentation and appressorial turgor pressure. Furthermore, we also found that the appressoria of the $\triangle C g C r z A$ mutant were defective in subsequent invasive hyphal development in $C$. gloeosporioides. These defects of invasive structure development in the $\triangle C g C r z A$ mutant may better explain why the mutant became nonpathogenic on its host plants. In M. ory$z a e$, the $\Delta M o c r z 1$ impaired appressorial turgor generation caused by the disruption of lipid metabolism in appressorium-mediated plant infection (Wilson and Talbot, 2009). Similar pathogenicity attenuation has been demonstrated in the $\Delta B c c r z 1$ mutant of $B$. cinerea, and the $\Delta P d c r z 1 \mathrm{mu}-$ 
tant of Penicillium digitatum, respectively (Schumacher et al., 2008; Zhang et al., 2013). These results indicated that CgCrzA and its orthologs may have a conserved role in fungal pathogenesis.

We demonstrated that the localization pattern of the $\mathrm{CgCrzA}$ is regulated by calcineurin and exogenous $\mathrm{Ca}^{2+}$. The CgCrzA functions as a transcriptional regulator plays important roles in pigmentation, CWI maintenance, invasive structure development, and pathogenicity of $C$. gloeosporioides. This study helped unveil the regulatory mechanisms that were critical for infection-related morphogenesis and pathogenicity of C. gloeosporioides. Further researches on determination of the downstream components of $\mathrm{CgCrzA}$ will facilitate further understanding of the pathogenic mechanism in $C$. gloeosporioides as well as other fungal pathogens.

\section{Acknowledgments}

This study was financially supported by the National Key R \& D Program of China (2017YFD0600102), National Nature Science Foundation of China (31870631), and Major Project of Jiangsu Province University Natural Science Research (16KJA220002).

\section{Electronic Supplementary Material}

Supplementary materials are available at The Plant Pathology Journal website (http://www.ppjonline.org/).

\section{References}

Araújo, L., Gonçalves, A. E. and Stadnik, M. J. 2014. Ulvan effect on conidial germination and appressoria formation of Colletotrichum gloeosporioides. Phytoparasitica 42:631-640.

Bulik, D. A., Olczak, M., Lucero, H. A., Osmond, B. C., Robbins, P. W. and Specht, C. A. 2003. Chitin synthesis in Saccharomyces cerevisiae in response to supplementation of growth medium with glucosamine and cell wall stress. Eukaryot. Cell 2:886-900.

Cao, H., Huang, P., Zhang, L., Shi, Y., Sun, D., Yan, Y., Liu, X., Dong, B., Chen, G., Snyder, J. H., Lin, F. and Lu, J. 2016. Characterization of 47 Cys2-His2 zinc finger proteins required for the development and pathogenicity of the rice blast fungus Magnaporthe oryzae. New Phytol. 211:1035-1051.

Chang, H. X., Miller, L. A. and Hartman, G. L. 2014. Melaninindependent accumulation of turgor pressure in appressoria of Phakopsora pachyrhizi. Phytopathology 104:977-984.

Chen, L., Tong, Q., Zhang, C. and Ding, K. 2019. The transcription factor $\mathrm{FgCrz} 1 \mathrm{~A}$ is essential for fungal development, virulence, deoxynivalenol biosynthesis and stress responses in Fusarium graminearum. Curr. Genet. 65:153-166.

Chen, W., Provart, N. J., Glazebrook, J., Katagiri, F., Chang, H. S., Eulgem, T., Mauch, F., Luan, S., Zou, G., Whitham, S. A., Budworth, P. R., Tao, Y., Xie, Z., Chen, X., Lam, S., Kreps, J. A., Harper, J. F., Si-Ammour, A., Mauch-Mani, B., Heinlein, M., Kobayashi, K., Hohn, T., Dangl, J. L., Wang, X. and Zhu, T. 2002. Expression profile matrix of Arabidopsis transcription factor genes suggests their putative functions in response to environmental stresses. Plant Cell 14:559-574.

Choi, J., Kim, Y., Kim, S., Park, J. and Lee, Y.-H. 2009. MoCRZ1, a gene encoding a calcineurin-responsive transcription factor, regulates fungal growth and pathogenicity of Magnaporthe oryzae. Fungal Genet. Biol. 46:243-254.

Cyert, M. S. 2003. Calcineurin signaling in Saccharomyces cerevisiae: how yeast go crazy in response to stress. Biochem. Biophys. Res. Commun. 311:1143-1150.

Dean, R., Van Kan, J. A., Pretorius, Z. A., Hammond-Kosack, K. E., Di Pietro, A., Spanu, P. D., Rudd, J. J., Dickman, M., Kahmann, R., Ellis, J. and Foster, G. D. 2012. The top 10 fungal pathogens in molecular plant pathology. Mol. Plant Pathol. 13:414-430.

Dichtl, K., Samantaray, S. and Wagener, J. 2016. Cell wall integrity signaling in human pathogenic fungi. Cell. Microbiol. 18:1228-1238.

Doering, T. L., Nosanchuk, J. D., Roberts, W. K. and Casadevall, A. 1999. Melanin as a potential cryptococcal defence against microbicidal proteins. Med. Mycol. 37:175-181.

Dubey, A. K., Barad, S., Luria, N., Kumar, D., Espeso, E. A. and Prusky, D. B. 2016. Cation-stress-responsive transcription factors SltA and CrzA regulate morphogenetic processes and pathogenicity of Colletotrichum gloeosporioides. PLoS ONE 11:e0168561.

Eisenman, H. C. and Casadevall, A. 2012. Synthesis and assembly of fungal melanin. Appl. Microbiol. Biotechnol. 93:931940.

Englbrecht, C. C., Schoof, H. and Böhm, S. 2004. Conservation, diversification and expansion of $\mathrm{C}_{2} \mathrm{H}_{2}$ zinc finger proteins in the Arabidopsis thaliana genome. BMC Genomics 5:39.

Fang, Y. L., Xia, L. M., Wang, P., Zhu, L. H., Ye, J. R. and Huang, L. 2018. The MAPKKK CgMck1 required for cell wall integrity, appressorium development, and pathogenicity in Colletotrichum gloeosporioides. Genes (Basel) 9:543.

Foster, A. J., Jenkinson, J. M. and Talbot, N. J. 2003. Trehalose synthesis and metabolism are required at different stages of plant infection by Magnaporthe grisea. EMBO J. 22:225235.

García, R., Bermejo, C., Grau, C., Pérez, R., Rodríguez-Peña, J. M., Francois, J., Nombela, C. and Arroyo, J. 2004. The global transcriptional response to transient cell wall damage in Saccharomyces cerevisiae and its regulation by the cell integrity signaling pathway. J. Biol. Chem. 279:15183-15195.

Garrett-Engele, P., Moilanen, B. and Cyert, M. S. 1995. Calcineurin, the $\mathrm{Ca}^{2+} /$ calmodulin-dependent protein phosphatase, is essential in yeast mutants with cell integrity defects and in 
mutants that lack a functional vacuolar $\mathrm{H}^{+}$-ATPase. Mol. Cell. Biol. 15:4103-4114.

Geoghegan, I., Steinberg, G. and Gurr, S. 2017. The role of the fungal cell wall in the infection of plants. Trends Microbiol. 25:957-967.

Gow, N. A. R., Latge, J.-P. and Munro, C. A. 2017. The fungal cell wall structure, biosynthesis, and function. Microbiol. Spectr. 5:FUNK-0035-2016.

He, F., Zhang, X., Mafurah, J. J., Zhang, M., Qian, G., Wang, R., Safdar, A., Yang, X., Liu, F. and Dou, D. 2016. The transcription factor VpCRZ1 is required for fruiting body formation and pathogenicity in Valsa pyri. Microb. Pathog. 95:101-110.

Huang, L., Kim, K.-T., Yang, J.-Y., Song, H., Choi, G., Jeon, J., Cheong, K., Ko, J., Xu, H. and Lee, Y.-H. 2019. A highquality draft genome sequence of Colletotrichum gloeosporioides sensu stricto SMCG1\#C, a causal agent of anthracnose on Cunninghamia lanceolata in China. Mol. Plant-Microbe Interact. 32:139-141.

Kim, S., Hu, J., Oh, Y., Park, J., Choi, J., Lee, Y.-H., Dean, R. A. and Mitchell, T. K. 2010. Combining ChIP-chip and expression profiling to model the MoCRZ1 mediated circuit for $\mathrm{Ca} /$ calcineurin signaling in the rice blast fungus. PLoS Pathog. 6:e1000909.

Kusuya, Y., Hagiwara, D., Sakai, K., Yaguchi, T., Gonoi, T. and Takahashi, T. 2017. Transcription factor Afmac1 controls copper import machinery in Aspergillus fumigatus. Curr. Genet. 63:777-789.

Langner, T. and Göhre, V. 2016. Fungal chitinases: function, regulation, and potential roles in plant/pathogen interactions. Curr. Genet. 62:243-254.

Lenardon, M. D., Munro, C. A. and Gow, N. A. 2010. Chitin synthesis and fungal pathogenesis. Curr. Opin. Microbiol. 13:416-423.

Levin, D. E. 2011. Regulation of cell wall biogenesis in Saccharomyces cerevisiae: the cell wall integrity signaling pathway. Genetics 189:1145-1175.

Li, B., Dong, X., Zhao, R., Kou, R., Zheng, X. and Zhang, H. 2019. The t-SNARE protein FgPep12, associated with FgVam7, is essential for ascospore discharge and plant infection by trafficking $\mathrm{Ca}^{2+}$ ATPase FgNeol between Golgi and endosome/vacuole in Fusarium graminearum. PLoS Pathog. 15:e1007754.

Madrid, M. P., Di Pietro, A. and Roncero, M. I. 2003. Class V chitin synthase determines pathogenesis in the vascular wilt fungus Fusarium oxysporum and mediates resistance to plant defence compounds. Mol. Microbiol. 47:257-266.

Martín-Udíroz, M., Madrid, M. P. and Roncero, M. I. 2004. Role of chitin synthase genes in Fusarium oxysporum. Microbiology (Reading) 50:3175-3187.

Pabo, C. O. and Sauer, R. T. 1992. Transcription factors: structural families and principles of DNA recognition. Annu. Rev. Biochem. 61:1053-1095.

Park, H.-S., Lee, S. C., Cardenas, M. E. and Heitman, J. 2019. Calcium-calmodulin-calcineurin signaling: a globally con- served virulence cascade in eukaryotic microbial pathogens. Cell Host Microbe 26:453-462.

Pusztahelyi, T. 2018. Chitin and chitin-related compounds in plant-fungal interactions. Mycology 9:189-201.

Ryder, L. S., Dagdas, Y. F., Mentlak, T. A., Kershaw, M. J., Thornton, C. R., Schuster, M., Chen, J., Wang, Z. and Talbot, N. J. 2013. NADPH oxidases regulate septin-mediated cytoskeletal remodeling during plant infection by the rice blast fungus. Proc. Natl. Acad. Sci. U. S. A. 110:3179-3184.

Schumacher, J. 2016. DHN melanin biosynthesis in the plant pathogenic fungus Botrytis cinerea is based on two developmentally regulated key enzyme (PKS)-encoding genes. Mol. Microbiol. 99:729-748.

Schumacher, J., de Larrinoa, I. F. and Tudzynski, B. 2008. Calcineurin-responsive zinc finger transcription factor $C R Z 1$ of Botrytis cinerea is required for growth, development, and full virulence on bean plants. Eukaryot. Cell 7:584-601.

Son, H., Seo, Y.-S., Min, K., Park, A. R., Lee, J., Jin, J.-M., Lin, Y., Cao, P., Hong, S.-Y., Kim, E.-K., Lee, S.-H., Cho, A., Lee, S., Kim, M.-G., Kim, Y., Kim, J.-E., Kim, J.-C., Choi, G. J., Yun, S.-H., Lim, J. Y., Kim, M., Lee, Y.-H., Choi, Y.-D. and Lee, Y.-W. 2011. A phenome-based functional analysis of transcription factors in the cereal head blight fungus, Fusarium graminearum. PLoS Pathog. 7:e1002310.

Soriani, F. M., Malavazi, I., da Silva Ferreira, M. E., Savoldi, M., Von Zeska Kress, M. R., de Souza Goldman, M. H., Loss, O., Bignell, E. and Glodman, G. H. 2008. Functional characterization of the Aspergillus fumigatus CRZ1 homologue, CrzA. Mol. Microbiol. 67:1274-1291.

Soulié, M. C., Perino, C., Piffeteau, A., Choquer, M., Malfatti, P., Cimerman, A., Kunz, C., Boccara, M. and Vidal-Cros, A. 2006. Botrytis cinerea virulence is drastically reduced after disruption of chitin synthase class III gene (Bcchs3a). Cell. Microbiol. 8:1310-1321.

Soulié, M.-C., Piffeteau, A., Choquer, M., Boccara, M. and VidalCros, A. 2003. Disruption of Botrytis cinerea class I chitin synthase gene Bcchs1 results in cell wall weakening and reduced virulence. Fungal Genet. Biol. 40:38-46.

Stathopoulos, A. M. and Cyert, M. S. 1997. Calcineurin acts through the $C R Z 1 / T C N 1$-encoded transcription factor to regulate gene expression in yeast. Genes Dev. 11:3432-3444.

Thompson, J. E., Fahnestock, S., Farrall, L., Liao, D. I., Valent, B. and Jordan, D. B. 2000. The second naphthol reductase of fungal melanin biosynthesis in Magnaporthe grisea. J. Biol. Chem. 275:34867-34872.

Wang, T., Ren, D., Guo, H., Chen, X., Zhu, P., Nie, H. and Xu, L. 2020. CgSCD1 is essential for melanin biosynthesis and pathogenicity of Colletotrichum gloeosporioides. Pathogens 9:141.

Wilson, R. A. and Talbot, N. J. 2009. Under pressure: investigating the biology of plant infection by Magnaporthe oryzae. Nat. Rev. Microbiol. 7:185-195.

Xu, J. R., Staiger, C. J. and Hamer, J. E. 1998. Inactivation of the mitogen-activated protein kinase Mps1 from the rice blast 
fungus prevents penetration of host cells but allows activation of plant defense responses. Proc. Natl. Acad. Sci. U. S. A. 95:12713-12718.

Xu, X., Wang, Y., Tian, C. and Liang, Y. M. 2016. The Colletotrichum gloeosporioides RhoB regulates cAMP and stress response pathways and is required for pathogenesis. Fungal Genet. Biol. 96:12-24.

Yang, J.-Y., Fang, Y.-L., Wang, P., Ye, J.-R. and Huang, L. 2018. Pleiotropic roles of $\mathrm{ChSat} 4$ in asexual development, cell wall integrity maintenance, and pathogenicity in Colletotrichum higginsianum. Front. Microbiol. 9:2311.

Yu, J.-H., Hamari, Z., Han, K.-H., Seo, J.-A., Reyes-Domínguez, Y. and Scazzocchio, C. 2004. Double-joint PCR: a PCRbased molecular tool for gene manipulations in filamentous fungi. Fungal Genet. Biol. 41:973-981.

Yu, X., Huo, L., Liu, H., Chen, L., Wang, Y. and Zhu, X. 2015. Melanin is required for the formation of the multi-cellular conidia in the endophytic fungus Pestalotiopsis microspora.
Microbiol. Res. 179:1-11.

Yun, Y., Zhou, X., Yang, S., Wen, Y., You, H., Zheng, Y., Norvienyeku, J., Shim, W.-B. and Wang, Z. 2019. Fusarium oxysporum $f$. sp. lycopersici $\mathrm{C}_{2} \mathrm{H}_{2}$ transcription factor FolCzfl is required for conidiation, fusaric acid production, and early host infection. Curr. Genet. 65:773-783.

Zhang, T., Xu, Q., Sun, X. and Li, H. 2013. The calcineurin-responsive transcription factor Crz1 is required for conidation, full virulence and DMI resistance in Penicillium digitatum. Microbiol. Res. 168:211-222.

Zhao, C., Jung, U. S., Garrett-Engele, P., Roe, T., Cyert, M. S. and Levin, D. E. 1998. Temperature-induced expression of yeast FKS2 is under the dual control of protein kinase $\mathrm{C}$ and calcineurin. Mol. Cell. Biol. 18:1013-1022.

Zhou, X., Li, G. and Xu, J.-R. 2011. Efficient approaches for generating GFP fusion and epitope-tagging constructs in filamentous fungi. Methods Mol. Biol. 722:199-212. 